\title{
Rejuvenating Smile with All-ceramic Crowns
}

\author{
${ }^{1}$ Kushal N Gandhi, ${ }^{2}$ Sabita M Ram, ${ }^{3}$ Naisargi P Shah
}

\begin{abstract}
Smile enhancement for a patient instills self-confidence by rendering an attractive smile. All-ceramic restorations have the potential to replicate the natural esthetics close to natural dentition, i.e., lacking in metal ceramic restorations. A young female patient unhappy with her smile was rehabilitated with all-ceramic restorations; thereby, the beauty of an individual within the functional and physiological functions could be restored. The final outcome with regard to esthetics and function was found to be good.
\end{abstract}

Keywords: All-ceramic restorations, Lithium disilicate, Smile enhancement.

How to cite this article: Gandhi KN, Ram SM, Shah NP. Rejuvenating Smile with All-ceramic Crowns. J Contemp Dent 2016;6(1):80-84.

\section{Source of support: Nil}

\section{Conflict of interest: None}

\section{INTRODUCTION}

Esthetics is of prime importance to patients, as it has a huge impact on the self-esteem. The desire to mimic natural tooth characteristics has made esthetic dentistry one of the areas of greatest demand.

Metal ceramic restorations have been the choice for more than three decades. They gained popularity for their predictable performance and reasonable esthetics. Despite their success, the demand for improved esthetics has led to the introduction of all-ceramic restorations. ${ }^{1,2}$ These restorations have the potential to replicate the appearance of the natural dentition. Significant developments in all-ceramic materials have created wonderful opportunities for the fabrication of life-like restorations that provide reliable and long-term results. Lithium disilicate, one of the high-end all-ceramic esthetic material, helps in achieving more esthetically pleasing restorations making it replace the time-tested metal ceramic restorations. ${ }^{3,4}$

\footnotetext{
${ }^{1}$ Postgraduate Student (3rd Year), ${ }^{2}$ Dean, Professor and Head, ${ }^{3}$ Professor

${ }^{1-3}$ Department of Prosthodontics, MGM Dental College and Hospital, Navi Mumbai, Maharashtra, India
}

Corresponding Author: Kushal N Gandhi, Postgraduate Student (3rd Year), Department of Prosthodontics, MGM Dental College and Hospital, Navi Mumbai, Maharashtra, India Phone: +919819235890, e-mail: drkushalng9@gmail.com
This case report presents the rehabilitation of a patient with prominent maxillary anterior teeth with lithium disilicate all-ceramic material restoration.

\section{CASE DESCRIPTION}

A 25-year-old female patient reported to the Department of Prosthodontics with a chief complaint of an unattractive smile due to prominent anterior teeth that restrained her from smiling. Patient had a history of orthodontic treatment 2 years back. She had a prominent crown on 21 . No relevant medical history was given by the patient.

On extraoral examination, patient had an ovoid facial form with a convex facial profile. Patient revealed a reverse smile with an average smile line displaying the gingival embrasures. Patient had an average lip length with thin upper lip and normal lower lip. The width of buccal corridor was adequate (Fig. 1).

On intraoral examination, the maxillary anterior teeth showed severe proclination that posed a negative effect on the smile of the patient. Patient had a prominent metal ceramic restoration with 21 and had an overjet of 5 to $6 \mathrm{~mm}$ and overbite of 2 to $3 \mathrm{~mm}$ from 12 to 22 . Canines were in proper position but discolored. Patient's upper dental midline coincided with facial midline, but upper dental midline did not coincide with lower dental

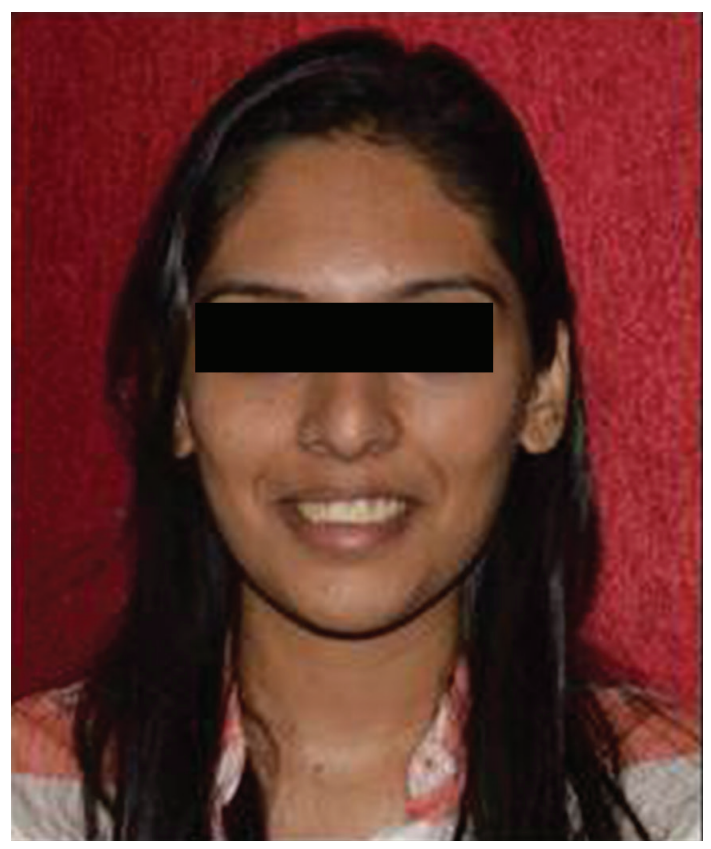

Fig. 1: Preoperative extraoral 

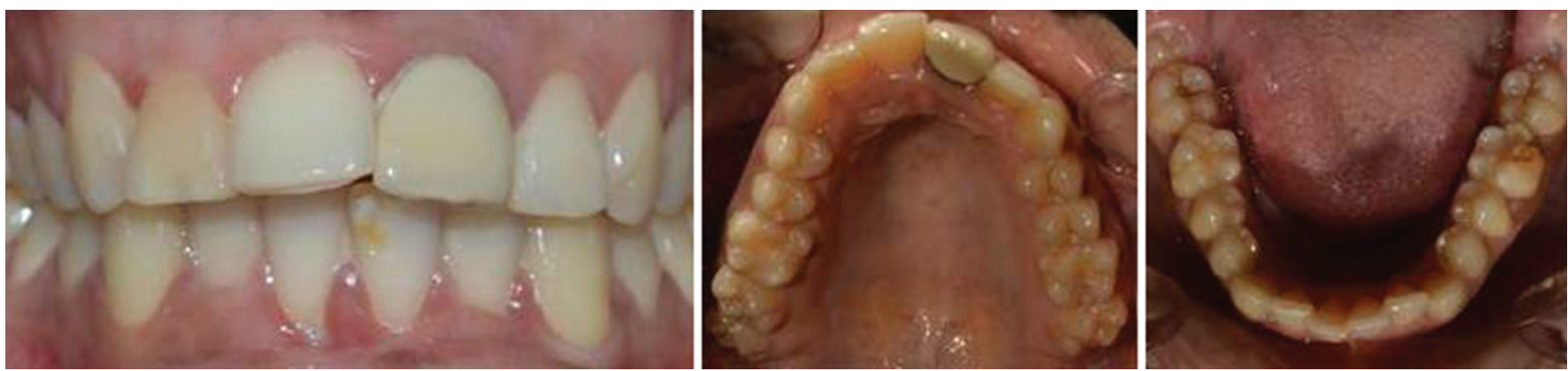

Fig. 2: Preoperative intraoral

midline. Patient had a canine-guided occlusion and patient's periodontal status was good (Fig. 2).

On radiographic examination, intraoral periapical radiograph (IOPA) with 21 showed root canal treatment that had proper obturation (Fig. 3); the orthopantomography (OPG) showed adequate bone and the dental status of the other teeth were good. Lateral cephalometric radiograph analysis concluded that the maxilla was more prominent (Fig. 4).

Diagnostic impressions were made in irreversible hydrocolloid and poured in dental stone to obtain a diagnostic cast that was analyzed. A diagnostic wax up was carried out considering the principles of smile designing. ${ }^{5-7}$ The position of the teeth was changed to get form and size to suit the patient and reduce the proclination to achieve the desired results. Two putty indices were made, one was used to aid in preparation and the other was used to make the provisionals. As the occlusion was canine-guided, the point contact was also

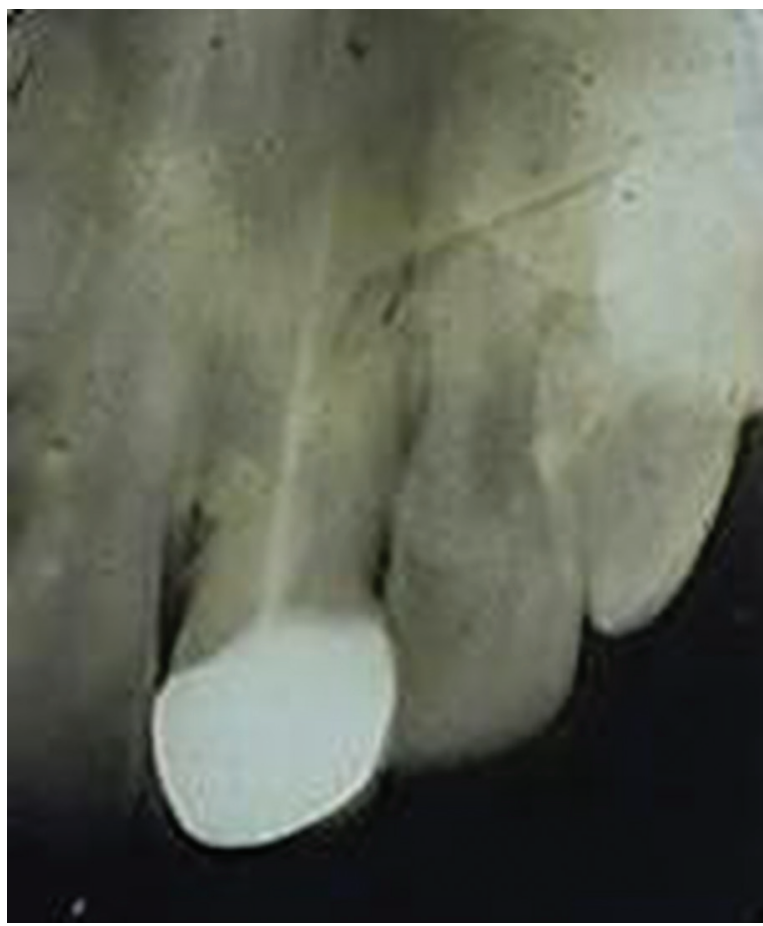

Fig. 3: Intraoral periapical showing RCT on 21 given on premolar teeth to reduce the load on maxillary canine restorations by carrying out enameloplasty of mandibular canines.

At first, scaling and polishing was carried out for both the arches. The old metal ceramic crown was cut away with carbide bur to protect the tooth structure. To correct the proclination for the desired results, it was planned to carry out endodontic treatment followed by i-post and composite core for 11, 12, 21, and 22 (Fig. 5). It was decided to restore the anterior esthetic zone using the lithium disilicate restorations. The consent of the patient was taken.

The shade of the mandibular teeth was selected as A1 using the Vita easy shade spectrophotometer. Tooth preparation was carried out with diamond point for incisors to receive full coverage lithium disilicate crowns to correct the severe proclination. All the incisors were prepared with an equicrevicular chamfer finish line.

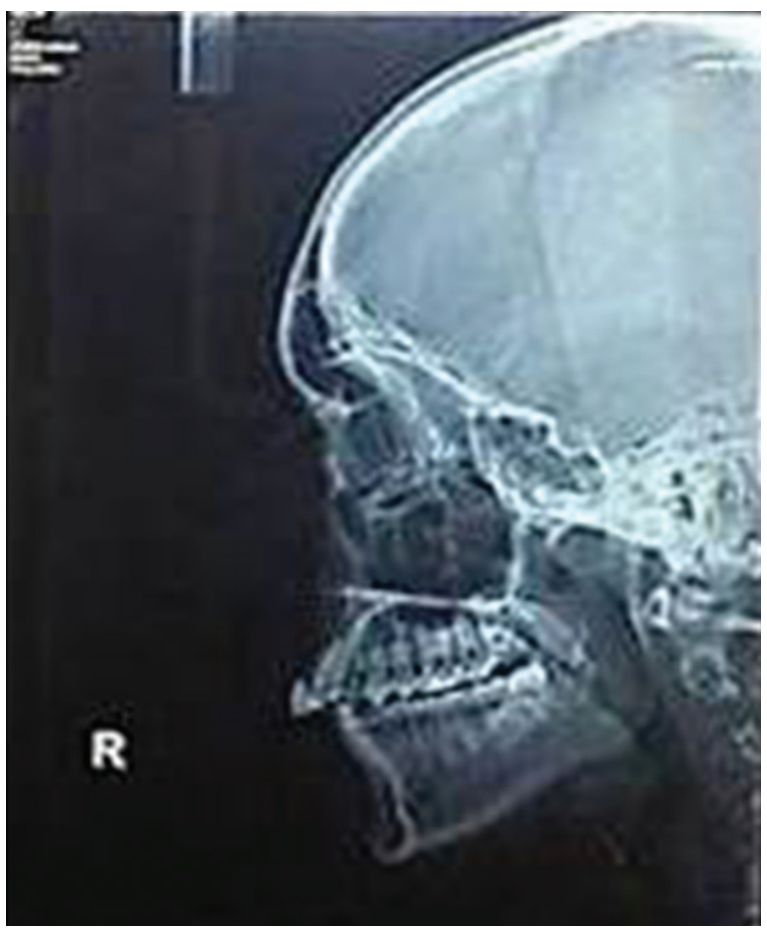

Fig. 4: Lateral cephalogram 


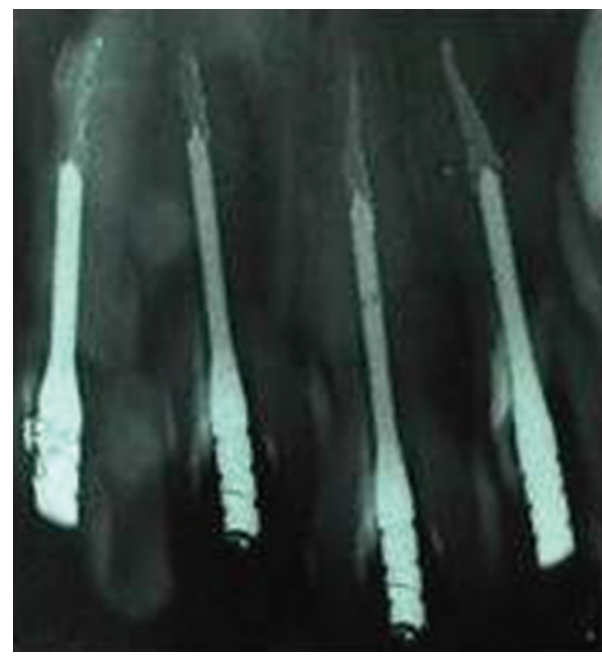

Fig. 5: Intraoral periapical with i-post

As maxillary canines were discolored and did not require repositioning, the preparation was carried out conservatively to receive porcelain laminates and preparations were kept in enamel at a depth of $0.5 \mathrm{~mm}$ using a depth-cutting diamond and a tapered diamond point (Fig. 6). The proximal preparation was extended beyond the contact area to avoid visibility of the tooth restoration junction. ${ }^{8}$ The silicon putty index was used to act as a preparation guide to verify the facial and incisal reduction as well as to confirm the uniformity in the thickness of porcelain (Fig. 7).

Mechanochemical gingival retraction was carried out with a knitted cord impregnated with $23 \%$ aluminum chloride. Once the preparation was completed, final impression was made using polyvinylsiloxane impression material by the two-stage double-mix technique (Fig. 8). Final impression was poured in type IV Gypsum to obtain a working cast using Pindex system.

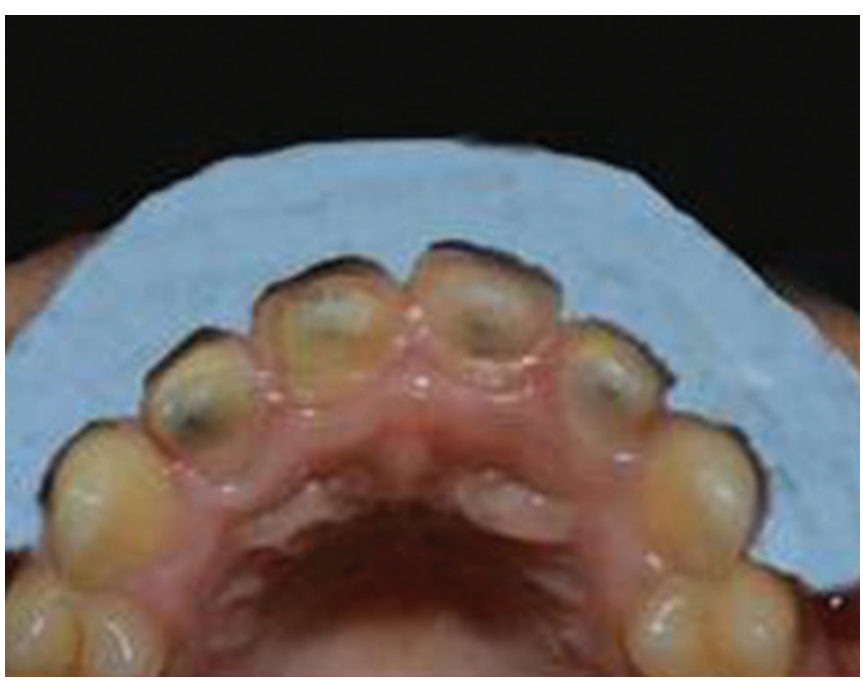

Fig. 7: Putty index to determine tooth reduction

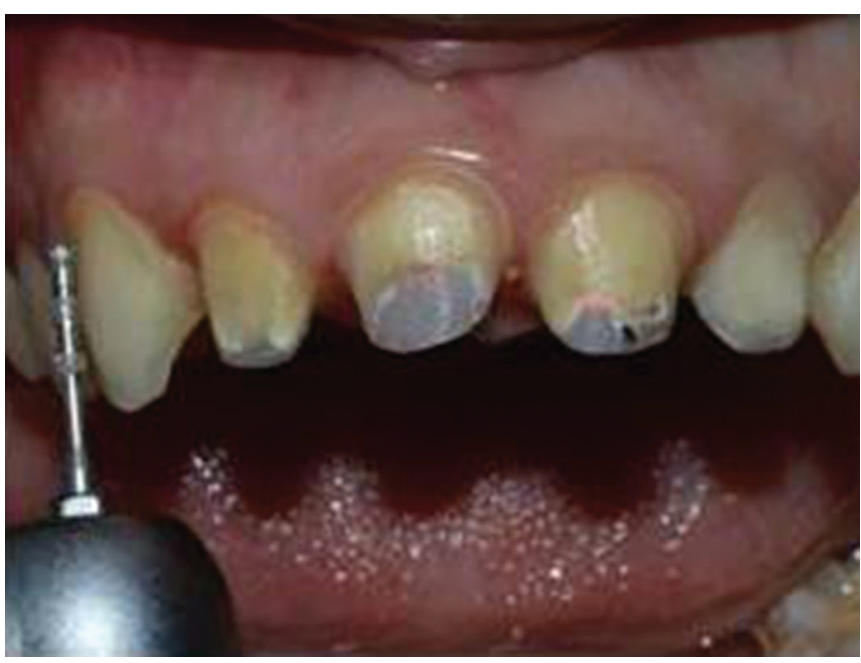

Fig. 6: Tooth preparation

Putty index was used to make provisional restorations with Protemp using direct technique immediately after the preparation of the teeth. The provisionals were assessed for shape and position of the teeth, whether it achieved the desired results (Fig. 9). This outcome was shown to the patient and her approval was taken so that this could be replicated in final restorations.

Porcelain lithium disilicate restorations for full coverage crowns and laminates were fabricated by the pressing technique using the correct shade ingot to obtain lithium disilicate restorations. The bisque trial of restorations were tried in for shade, fit, marginal adaptation, shape, size, symmetry, contacts, and the amount of visibility of the restoration at rest position and when the patient smiles. The occlusion was checked in centric and eccentric position and any interferences were removed. Patient's approval was obtained at the time of try-in. The final glazing was carried out.

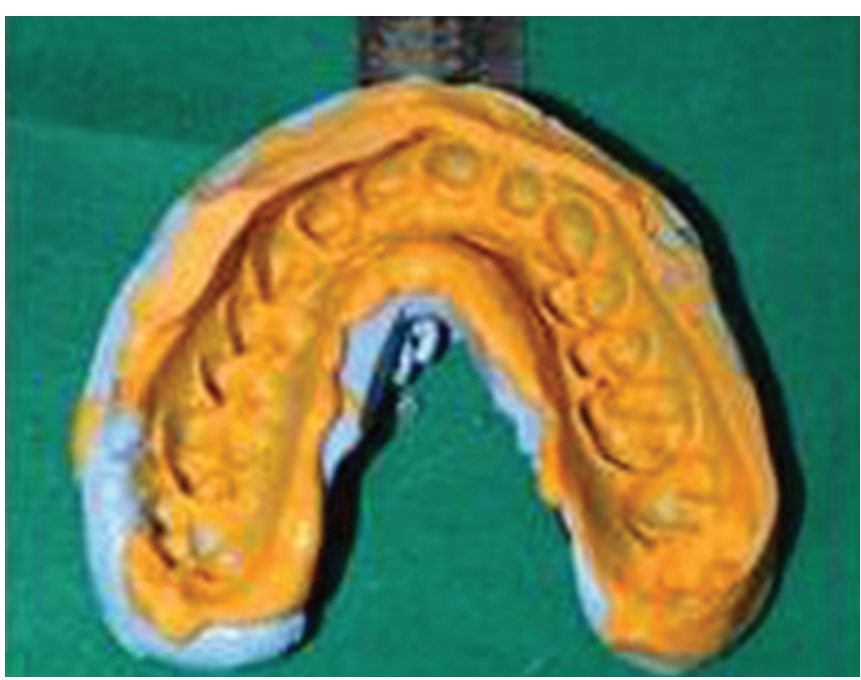

Fig. 8: Final impression 


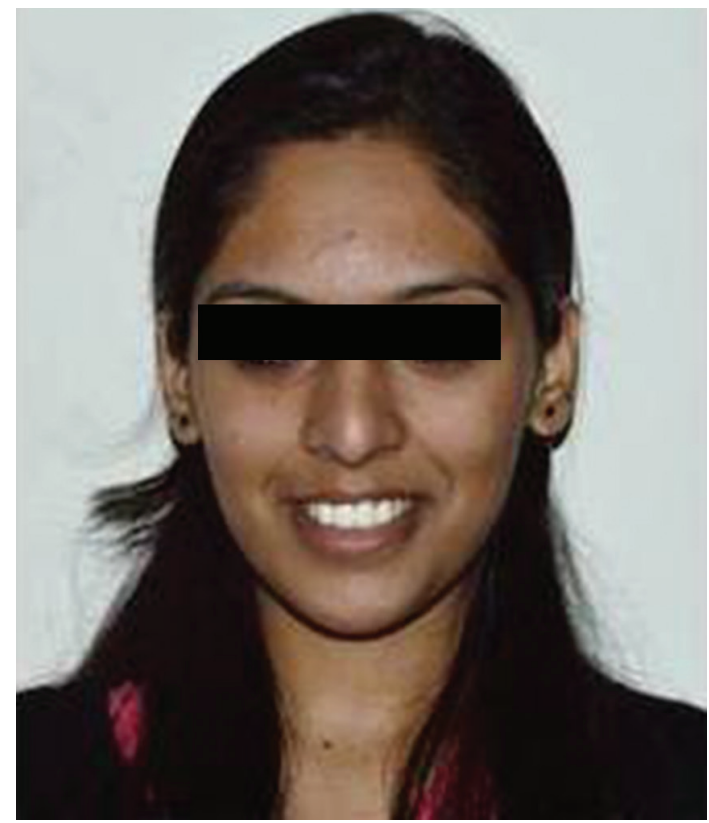

Fig. 9: Provisionals

At the luting appointment, the restorations were arranged denoting the position of the tooth in the arch to avoid incorrect placement. The procedure for luting was performed first on central incisors followed by lateral incisors and then the canines. Dual cure luting agent Rely X U200 [3M ESPE] was used for luting.

The lithium disilicate restorations were etched with $10 \%$ hydrofluoric acid for 20 seconds. After etching, they were washed thoroughly using liberal amount of water. On drying, a coat of silane coupling agent was applied for 60 seconds.

The prepared teeth were etched using $37 \%$ phosphoric acid for 15 seconds on incisors because it was in dentin and 30 seconds for canines as the preparation was in enamel, they were washed and air-dried. Then bonding agent was applied and light cured for 10 seconds on all prepared teeth. The restorations were spot cured for 5 seconds initially. Excess cement was removed with explorer and then complete curing was done for 20 seconds. The patient was satisfied with her new smile line and excellent alignment of the anterior teeth (Figs 10 to 12).

\section{DISCUSSION}

Proclined anterior teeth can be treated with orthodontic treatment; however, orthodontic relapse had taken place and therefore, repeated orthodontic treatment was not acceptable to the patient. Hence, it was planned to treat her with all-ceramic crowns to reposition the proclination.

In the present case, treating the patient only with allceramic crowns without any endodontic treatment was

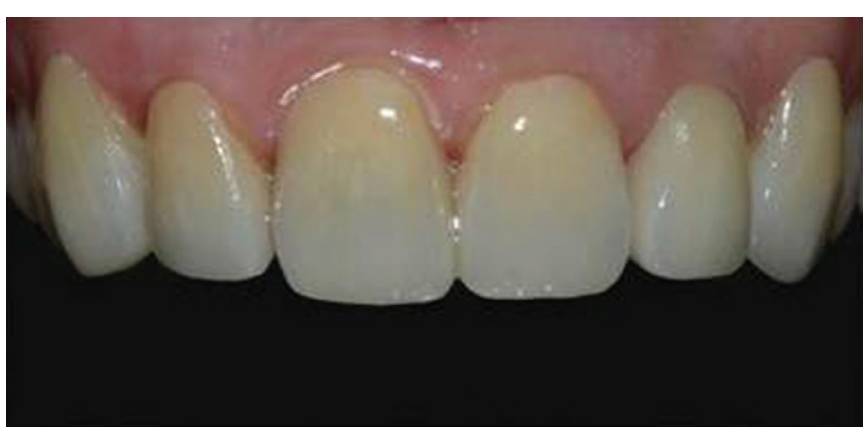

Fig. 10: Postoperative intraoral

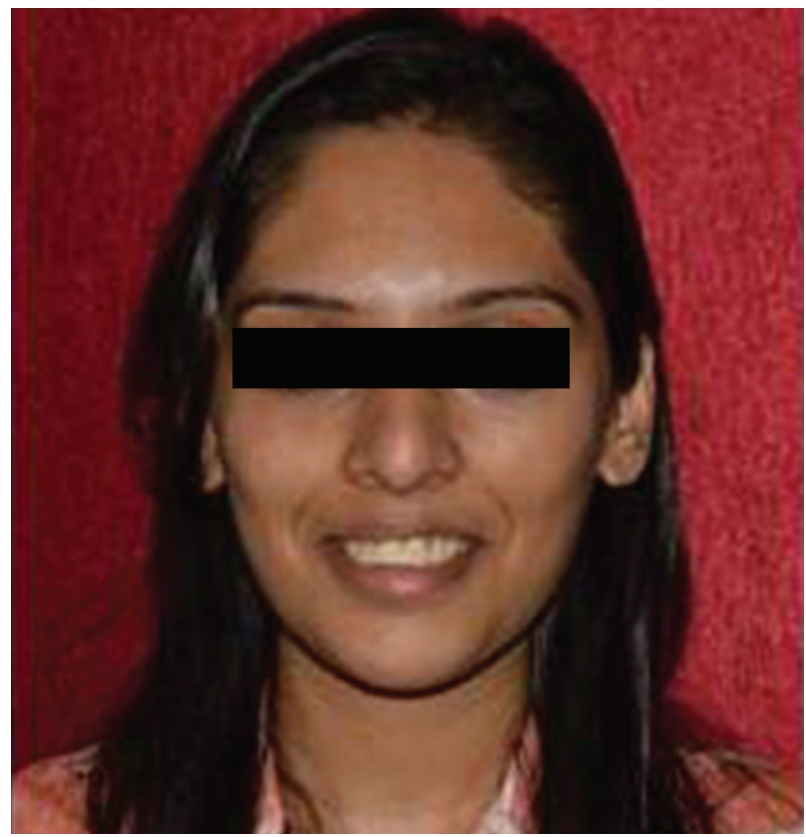

Fig. 11: Preoperative extraoral

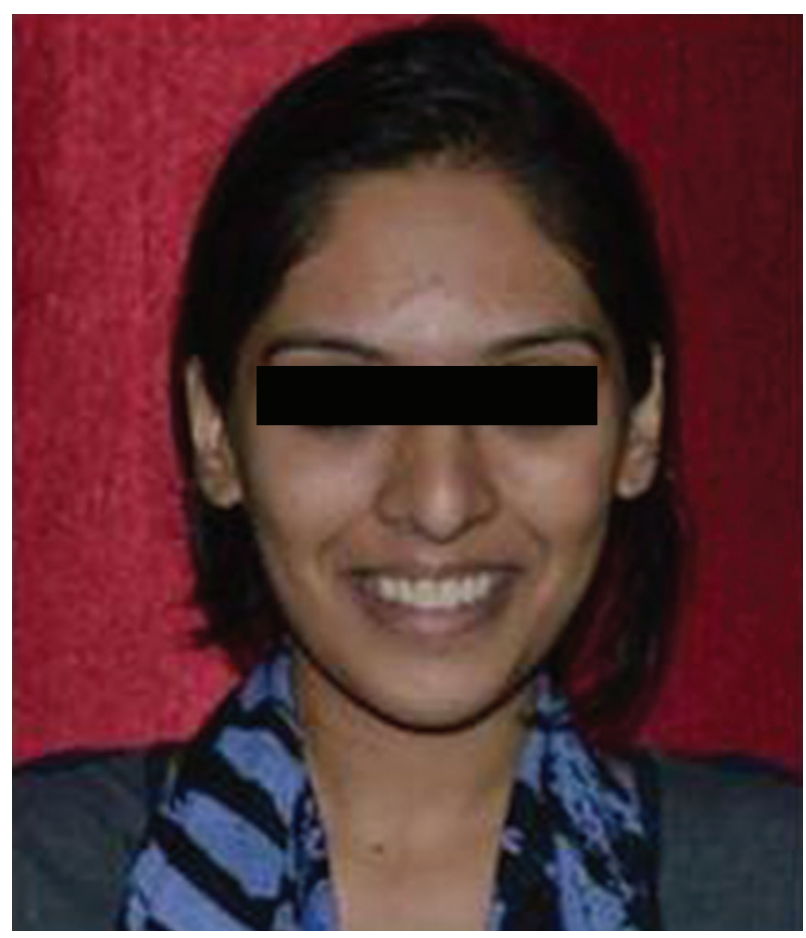

Fig. 12: Postoperative extraoral 

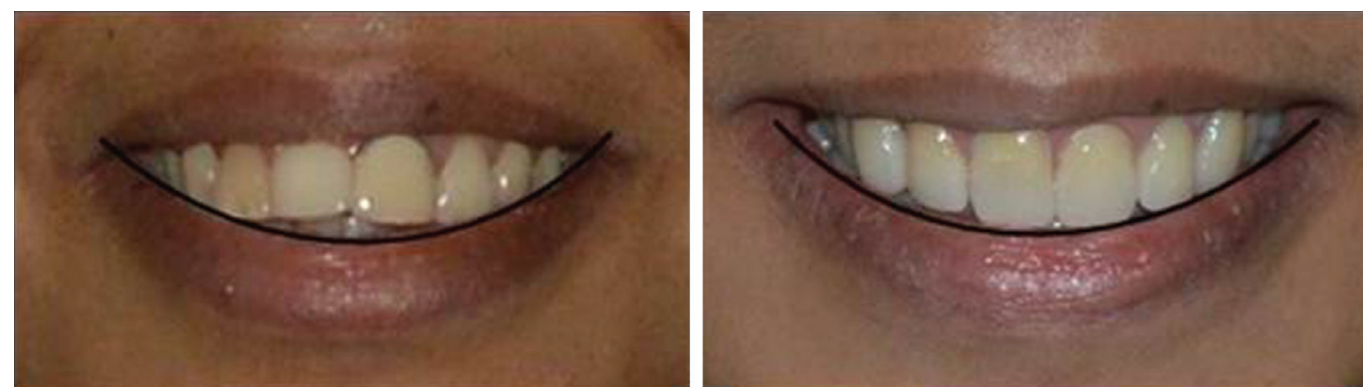

Fig. 13: Smile curve
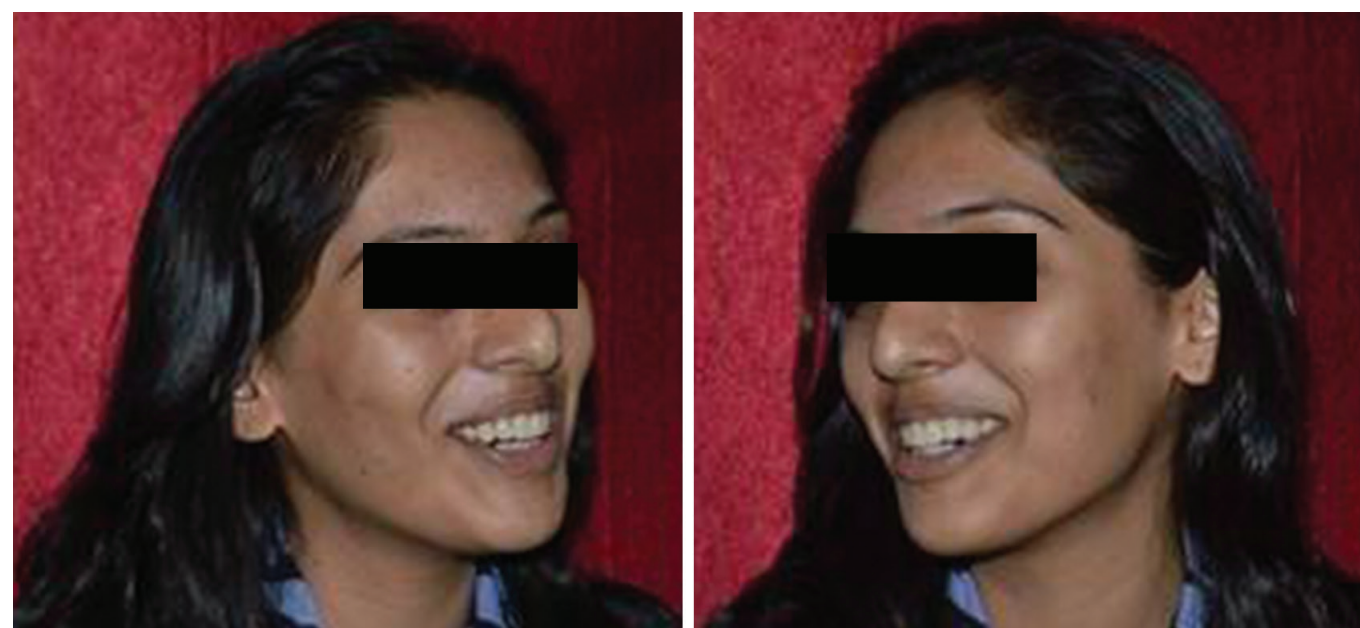

Fig. 14: Postoperative extraoral

not sufficient; intentional root canal treatment of maxillary incisors was carried to change the alignment of the teeth. As the proclination was not severe, it could be managed by prefabricated i-post with composite build-up; therefore, one-piece cast post and core was not needed in this case. Canines were treated conservatively with porcelain laminates, as they were discolored and did not require repositioning. The proclination of the teeth was corrected changing the reverse smile into a convex smile making the smile esthetically more pleasing (Fig. 13).

\section{CONCLUSION}

This is an interesting case wherein all-ceramic restorations were employed to optimally rejuvenate an unesthetic smile. Although the patient had orthodontic treatment that relapsed, this could be treated with prosthetic rehabilitation using all-ceramic restorations. The patient was more confident with the esthetically pleasing outcome and did not experience any phonetic problems resulting from the correction of the tooth alignment. The new smile of the patient was satisfactory with excellent esthetic appearance (Fig. 14).

Detailed planning, correct selection of dental materials, and quality communication with the prosthetic technician contributed to a harmonious smile and the evident satisfaction of both patient and dental professionals (Fig. 13).

\section{REFERENCES}

1. Kelley JR, Benetti P. Ceramic materials in dentistry. Historical evaluation and current practice. Aust Dent J 2011 Jun;56 (Suppl 1):84-96.

2. Hatai Y. Extreme masking: achieving predictable outcomes in challenging situations with lithium disilicate bonded restorations. Int J Esthet Dent 2014 Sum;9(2):206-222.

3. Ritter RG, Rego NA. Material considerations for using lithium disilicate as a thin veneer option. J Cosmetic Dent 2009; 25(3):111-117.

4. Soares PV, Spini PHR, Carvalho VF, Souza PG, Gonzaga $\mathrm{RCQ}$, Tolentino AB, Machado AC. Esthetic rehabilitation with laminated ceramic veneers reinforced by lithium disilicate. Quintessence Int 2014 Feb;45(2):129-133.

5. Davis NC. Smile design. Dent Clin N Am 2007 Apr; 51(2): 299-318.

6. Bhuvaneswaran M. Principles of smile design. J Conserv Dent 2010 Oct;13(4):225-232.

7. Ahmad I. Geometric considerations in anterior dental esthetics: restorative principles. Pract Periodontics Aesthet Dent 1998 Sep;10(7):813-822.

8. Gürel G. Predictable and precise tooth preparation techniques for porcelain laminate veneers in complex cases. Int Dent SA 2007;9(1):30-40. 